\title{
Performance Evaluation in Iris Recognition and CBIR System based on Phase Congruency
}

\author{
Pravin S. Patil \\ North Maharashtra University \\ Umavinagar, Jalgaon \\ Maharashtra, India
}

\author{
S. R. Kolhe \\ North Maharashtra University \\ Umavinagar, Jalgaon \\ Maharashtra, India
}

\author{
R. V. Patil \\ BSD College of Engineering \\ Dhule, Mahrashtra \\ India
}

\author{
P. M. Patil \\ RMD Sinhghad Technical Institutes Campus \\ Warje, Pune \\ Maharashtra, India
}

\begin{abstract}
Content based image retrieval is an important research area in image processing, with a vast domain of applications like recognition systems i.e. face, finger, iris biometric etc. It retrieves the similar type of images from repository of images based on users query. To retrieve similar images, color, and texture or shape features need to be extracted from the images and stored in the feature database. The color, texture or shape features of query image are compared with the features of images in the database. This comparison is performed using color, texture or shape distance metrics. Phase Congruency is applied to the input image for feature extraction. Advantage of the Phase Congruency method is its insensitivity to variations in image illumination and contrast. Like CBIR, the methods for iris recognition mainly focus on feature representation and matching. We use phase congruency to generate iris feature vector. Phase Congruency can be calculated using Log Gabor wavelets. The goal of this paper is to evaluate the performance of CBIR system and iris recognition that use Phase Congruency. To evaluate performance in CBIR System, precision and recall measures are used. In order to evaluate iris recognition system, recognition rate measure is used and compared with existing methods. Results of performance evaluation are discussed in paper.
\end{abstract}

\section{General Terms}

Security, Biometrics, CBIR, Iris Recognition

\section{Keywords}

Phase Congruency, Gabor Wavelet, Log Gabor Wavelet, Euclidean Distance, Hamming Distance.

\section{INTRODUCTION}

CBIR or Content Based Image Retrieval is the retrieval of similar images from the repository of images based on features such as colour, texture or shape [1], [2], [6], [7]. Traditional methods of image indexing that assign a keyword or number to an image with a categorized description have proved to be insufficient and time consuming. Such system is not CBIR. In CBIR, color, texture or shape features of each image in the database are extracted and compared to the features of the query image [1], [2].

Like CBIR, in biometrics systems images used as patterns (e.g. fingerprint, face, iris, hand etc.) are also represented by feature vectors [11], [12], [7]. CBIR and iris recognition, although similar in principle, using many of the same image analysis/statistical tools. Iris recognition works with an existing database of eye images and is primarily a statistical matching problem. One can argue that biometrics system is a particularly well defined sub-set of CBIR. Another distinction between recognition and retrieval, recognition decides whether or not an observed object matches rather than ranking the images according to their measured similarity to a query example [7].

Feature extraction is most critical in both retrieval and recognition systems because the particular features made available for matching directly influence the efficiency of the classification task [2], [7], [9]. Main reason for performing feature extraction is to reduce the computational complexity and dimensional feature space. Various algorithms have been applied for feature extraction. In recent years, Phase Congruency has been used for feature extraction in CBIR and iris recognition systems [3], [4], [5], [13]. We utilized a technique based on the phase congruency of the Fourier transform components for feature extraction. Phase Congruency is a dimensionless quantity that is invariant to changes in image brightness or contrast [14], [15], [16].

The remainder of this paper is organized as follows: Section 2 and 3 briefly discuss typical CBIR and Iris Recognition system. A detailed description of the feature extraction based on Phase Congruency is given in Section 4. Section 5 reports experiments and also discuss performance issues in CBIR and Iris Recognition system. Section 6 concludes this paper.

\section{TYPICAL CBIR SYSTEM}

In typical CBIR systems (Fig. 1), features of the images in the database are extracted and stored in the feature database [1], [2]. To retrieve similar set of images, user inputs query image to the retrieval system. The system then extracts feature vector from query image. The similarities between the feature vectors of the query image and those of the images in the database are then calculated using distance metrics and results of the retrieval are presented to the user with the help of an indexing scheme.

CBIR involves two steps. The first step extracts color, texture or shape features. The second step discover similarities between the feature vectors of the query image and those of the images in the database

CBIR applications are automatic face recognition systems, iris recognition system, used for crime preventions and access privileges. It is also used in a database of medical images to help diagnosis by identifying similar past cases [1], [2].

The color, texture and shape feature descriptors are mainly used for feature extraction. 


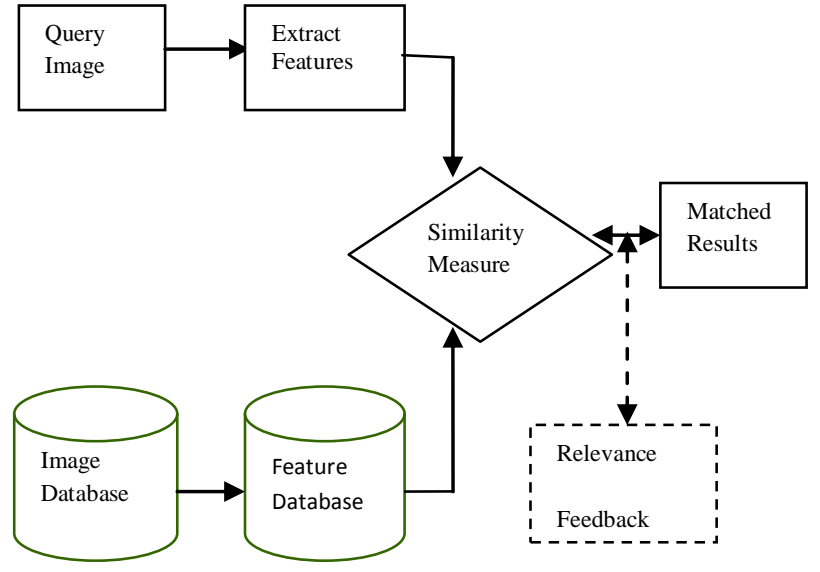

Figure 1. Typical CBIR System

\subsection{Color Features}

Color is significant dimension of human visual perception used to distinguish and recognize visual information. The key method of representing color information of images in CBIR systems is through color histograms .

\subsection{Texture Features}

Texture is property of all surfaces that describes visual patterns, each having properties of homogeneity such as bricks, leaves, fabrics, flowers, etc. It provides information about the structural arrangement of the surface. In short, it is a feature that describes the physical composition of a surface.

\subsection{Shape Features}

Shape can be defined as an outline or contour of an object. It permits an object to be distinguished from its background by its outline. Shape feature representations can be boundarybased or region based

\section{TYPICAL IRIS RECOGNITION SYSTEM}

A typical iris recognition system is schematically shown in Fig. 1. A typical iris recognition system often includes 1) Iris capturing; 2) Preprocessing; 3) Feature extraction and 4) Feature matching steps [18], [19],

A captured iris image contains not only the region of interest (iris) but also some 'unuseful' parts (e.g. eyelid, pupil etc.). So, the image cannot be used directly without preprocessing. In addition, a change in the camera-to-face distance may result in the possible variation in the size of the same iris [19], [20], [21] Furthermore, the brightness is not uniformly distributed because of non-uniform illumination [14], [24]. For the purpose of recognition, the original image needs to be preprocessed. The preprocessing of iris image includes four aspects: localization, normalization, enhancement, denoising, and the selection of iris valid areas. A detailed description of preprocessing methods is given in [8], [19].

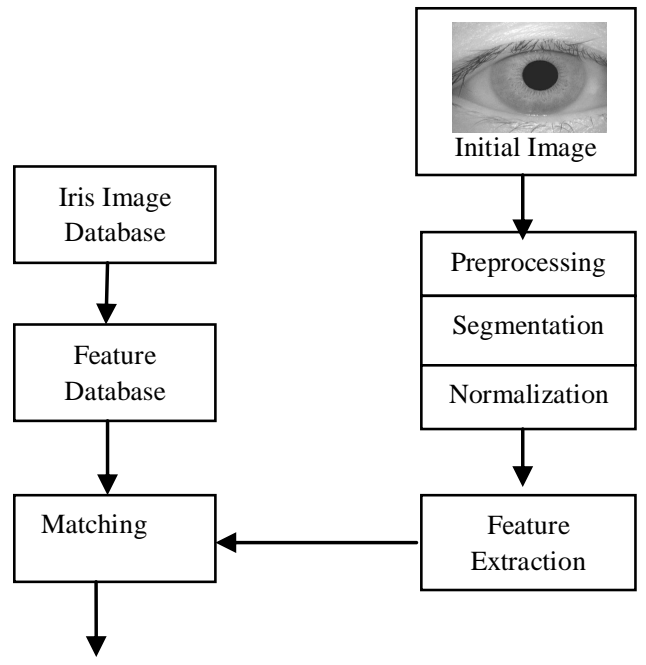

Decision

Figure 2. Typical Iris Recognition System

Iris feature extraction is the most important step in iris recognition, which determines directly the value of iris characteristics in actual application [19]. Much works has been reported by researchers till now [21], [24], [27], [29], [35]. John G. Daugman, a Professor of Cambridge University [1], [19], [20] proposed a typical and successful iris recognition system that makes use of a decomposition derived from application of two dimensional versions of Gabor filters to the image data to extract its phase information. Here we use phase congruency to represent iris features. Phase congruency is invariant to changes in image intensity or contrast.

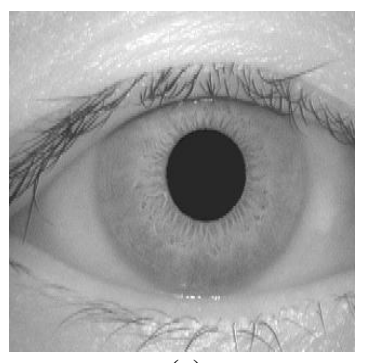

(a)

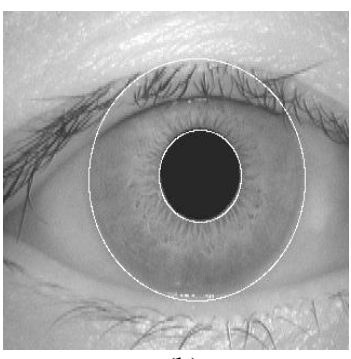

(b)

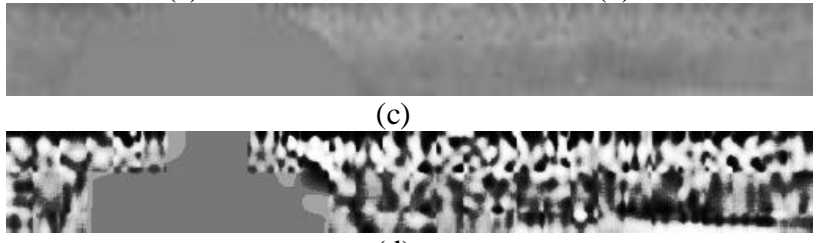
(d)

Figure 3. Preprocessing of Iris Image

(a) Original Eye Image (b) Image after Iris Localization (c) Normalization of Iris Image (d) Enhanced iris image

\section{FEATURE EXTRACTION USING PHASE CONGRUENCY}

Phase congruency is a novel feature detector [14], [15]. It detects edge and step responses. Its characteristics include invariance to lighting variation within images and invariance to magnification. Local energy model, postulates that features are perceived at point in an image where Fourier components are in phase. Morrone and Owens [16] developed this model. Phase congruency values are high at edge points and at 
boundary of object. So Phase congruency can be used as a feature detector in CBIR systems for extracting shape information from image [3], [4], [5].

Peter Kovesi proposed a method to calculate the phase congruency via logarithmic Gabor wavelets [10], [14], [15]. Log Gabor wavelets are used because they can cover large frequency space while still maintaining a zero DC component in the even symmetric filter. Filters are constructed in the frequency domain using a polar co-ordinate system. The filter has two components radial component and angular component [14]. Two components are multiplied to construct the overall filter. To calculate phase congruency image convolution is done with a set of $\log$ Gabor wavelets at different orientations and at different scales. 1-D log Gaussians in the radial direction has a transfer function of the form

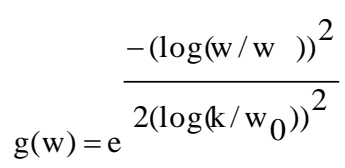

Where $w_{0}$ is the centre frequency of filter. To obtain constantshape ratio filters the term $k / w_{0}$ must also be held constant for varying $w_{0}$.

The Gaussian cross-section in the angular direction is defined as

$G(\theta)=\frac{\left(\theta-\theta_{0}\right)^{2}}{2 \sigma_{\theta}^{2}}$

Where $\theta_{\mathrm{o}}$ is the orientation angle of the filter, and $\sigma_{\mathrm{o}}$ is the standard deviation of the Gaussian function in the angular direction. Then at each location in the image, calculate energy $E(x)$ in each orientation, subtract the estimated noise effect To to eliminate spurious responses to noise, apply the weighting for frequency spread $W o(x)$, and form the sum over all orientations. This sum of energies is then normalized by dividing by the sum over all orientations and scales of the amplitudes of the individual wavelet responses at that location in the image. This produces the following equation for 2-D phase congruency:

$$
\begin{aligned}
& \mathrm{E}_{\mathrm{no}}=\mathrm{A}_{\mathrm{no}} \nabla \varphi_{\mathrm{no}}(\mathrm{x}) \\
& P C(x)=\frac{\sum_{o} \sum_{n}\left\lfloor E_{n o}(x)-T\right\rfloor}{\sum_{o} \sum_{n} A_{n o}(x)+\varepsilon}
\end{aligned}
$$

The phase deviation function is defined as,

$$
\Delta \phi_{n o}(x)=\cos \left(\phi_{n}(x)-\overline{\phi(x)}\right)-\mid \sin \left(\phi_{n}(x)-\overline{\phi(x)} \mid\right.
$$

The Compensation of noise $\mathrm{T}$ is defined as

$$
\mathrm{T}=\mu_{\mathrm{R}}+\mathrm{k} \sigma_{\mathrm{R}}
$$

Where $\mu_{R}$ and $S_{R}$ are the mean and variance of Rayleigh distribution. The Weighting function is defined

$$
\mathrm{W}(\mathrm{x})=\frac{1}{1+\mathrm{e}^{\mathrm{g}(\mathrm{c}-\mathrm{s}(\mathrm{x}))}}
$$

$$
s(x)=\frac{1}{N}\left(\frac{\sum_{n} A_{n}(x)}{\varepsilon+A_{\max }(x)}\right)
$$

Where $N$ is the number of scales, $\operatorname{Amax}(x)$ is the amplitude of the filter pair having maximum response at position $x, e$ is a small constant to avoid division by zero, $c$ is the cutoff value of filter response spread below which phase congruency values become penalized
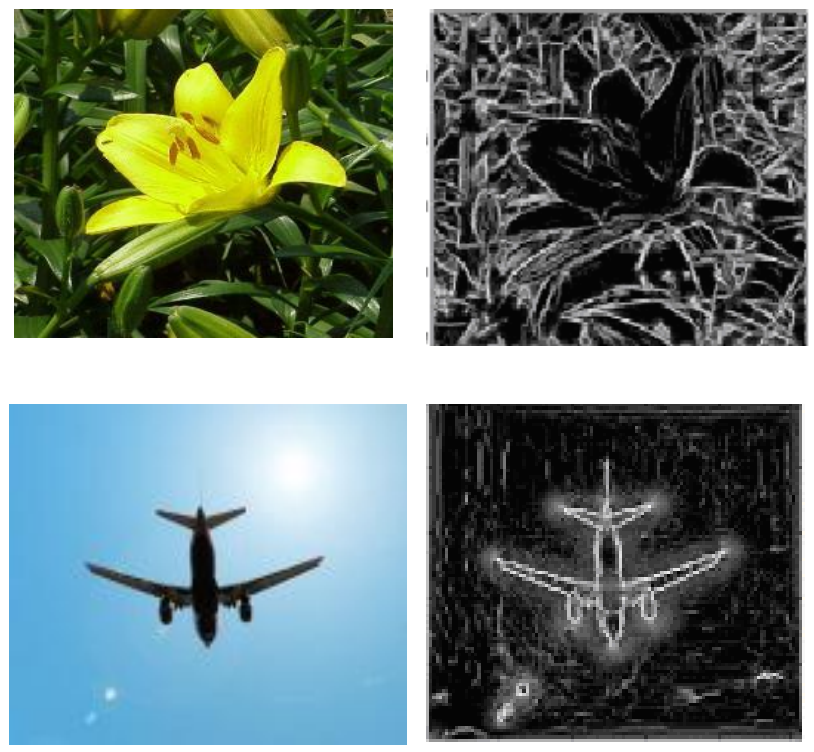

Figure 4. Original Image and Corresponding phase Congruency image

\section{EXPRIMENTAL RESULTS AND PERFORMANCE EVALUATION}

To implement CBIR System, We store 100 images on a computer. MATLAB image processing tools are used to implement system. We use phase congruency to extract shape features of query image and images in database as proposed in [3], [4]. Euclidean distance is used to compare Phase Congruency features of query image and images in database. To retrieve similar set of images, Euclidean distance is calculated between the phase congruency map of query image and every image in the database using following equation.

$$
d(q, d)=\sqrt{\left(P C_{q}-P C_{d}\right)^{2}}
$$

This process is repeated until all the images in the database have been compared with the query image. Upon completion of the Euclidean distance algorithm, we have an array of Euclidean distances, which is then sorted. The ten topmost images are then displayed as a result of the search.

After obtaining results, Performance evaluation of CBIR system is done. For Performance evaluation, we employed precision, recall and retrieval efficiency measures

Evaluation of image retrieval performance is a crucial problem in CBIR [1], [2], [25]. Many different methods for measuring performance of a system have been developed and used by researchers. The determination of relevant and nonrelevant images for a given query is one of the most time consuming tasks. The most common evaluation measures used in image retrieval are precision and recall. Researchers 
are familiar with precision and recall can extract information from them without interpretation problem [25].

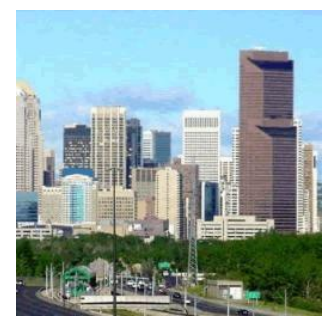

Figure 5.Query Image Building

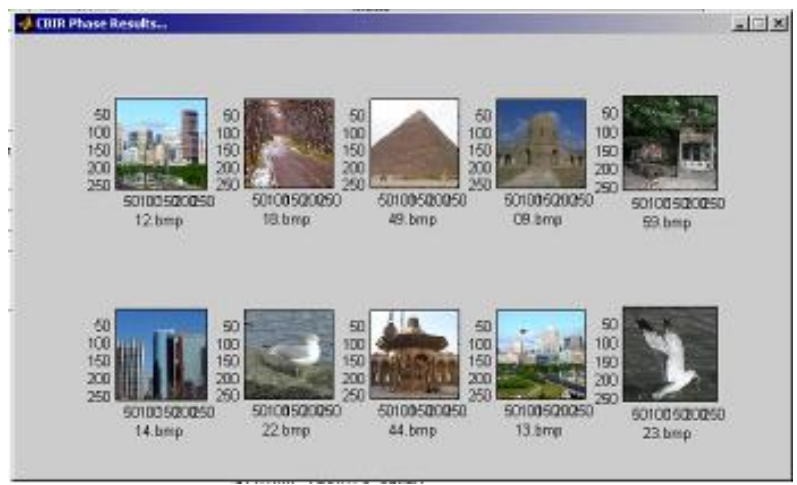

Figure 6. Phase Congruency Based CBIR System : Retrieval Results for Query Image Building

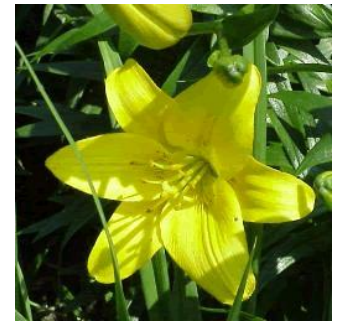

Figure 7. Query Image : Flower

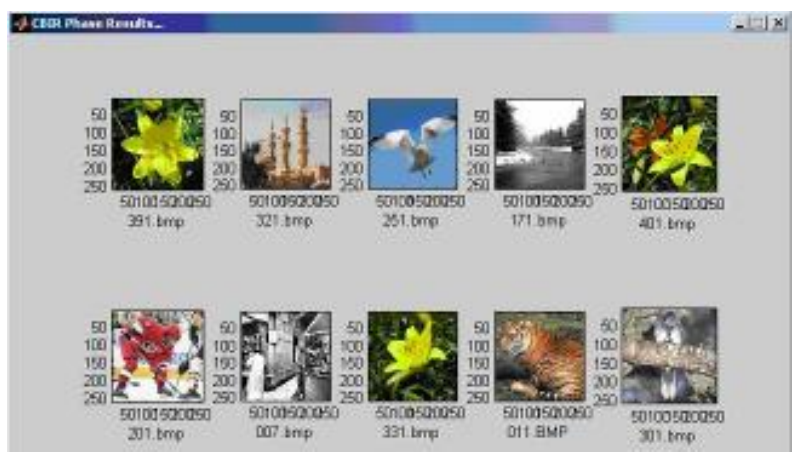

Figure 8. Phase Congruency Based CBIR System : Retrieval Results for Query Image Flower

$$
\begin{aligned}
& \text { Precision }=\frac{\text { No of relevantImages Retrieved }}{\text { Total noof Images Retrieved }} \\
& \text { Recall }=\frac{\text { Noof RelevantImages Retrieved }}{\text { Total Noof RelevantImages in database }}
\end{aligned}
$$

We have used precision and recall for evaluation of CBIR system. Precision depends on number of relevant images for a given query in database. If number of relevant images for a given query is less in database then this value will be low
[25]. Precision and recall value lies between 0 and 1 . To evaluate performance of CBIR systems we used small database of 100 images. We can make recall 1 simply by retrieving all images. We have calculated recall after retrieval of 10 images from database. Recall for different type of images found 0.66 to 1 . Number of relevant images from 10 retrieved images found to be 66 to 100 percent.

Alternate measures used for performance evaluation in CBIR System are error rate and retrieval efficiency [25]. Error rate is common used in face recognition for performance evaluation. Error rate is the ration of number of non-relevant images retrieved to the total number of images retrieved. Retrieval efficiency is the ratio of number of relevant images retrieved to the total number of images in database. If the number of images retrieved is lower or equal to the number of relevant images, this value is precision, otherwise it is recall. Recall, precision, error rate and retrieval efficiency are calculated for different types of query images as shown in Table 1 .

Table 1. Performance Evaluation for different Query Images

\begin{tabular}{|l|l|l|l|l|}
\hline Image & Precision & Recall & $\begin{array}{l}\text { Error } \\
\text { Rate }\end{array}$ & $\begin{array}{l}\text { Retrieval } \\
\text { Efficiency }\end{array}$ \\
\hline Flower & 0.5 & 0.75 & 0.116 & 0.5 \\
\hline Building & 0.66 & 1 & 0.116 & 0.66 \\
\hline Pyramid & 0.33 & 0.66 & 0.133 & 0.33 \\
\hline $\begin{array}{l}\text { Natures } \\
\text { Scene } \\
\text { (Snow fall } \\
\text { Road) }\end{array}$ & 0.33 & 1 & 0.133 & 0.33 \\
\hline
\end{tabular}

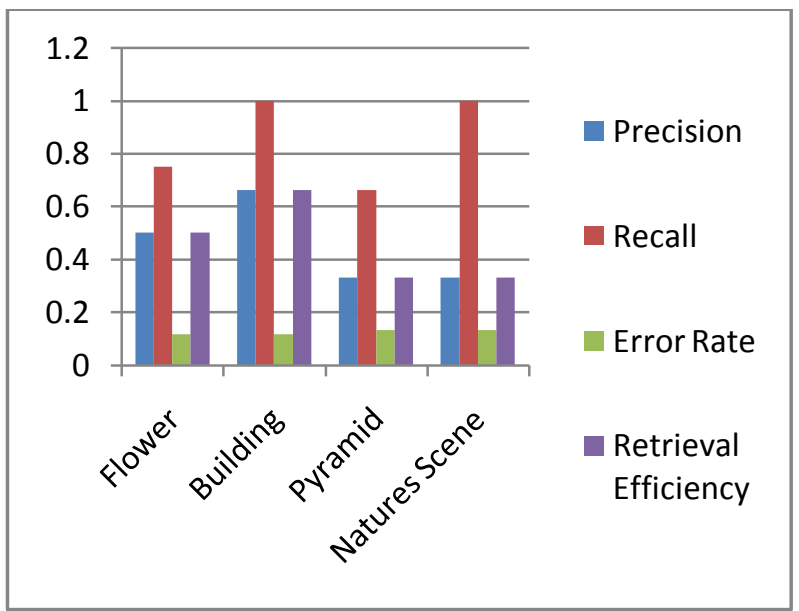

Figure 9. Precision, Recall Vs Query Images

Evaluating the performance of iris recognition algorithms is a difficult issue [26], [33]. For the purpose of evaluation; we implement iris recognition method according to the published papers [13], [24]. To compare their performance, we construct an iris image database named CASIA Iris Database. We use images of eyes from 30 persons, and every person has 10 images of eyes. MATLAB image processing tools were used to implement system. We use the usual methods to locate and normalize iris regions, and use phase congruency mentioned above to extract the feature. Phase congruency is calculated using Log Gabor wavelets. We used Hamming Distance similarity measures to measure the similarity of iris features. 


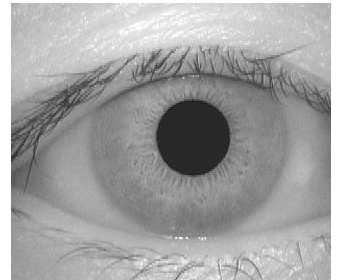

(a)

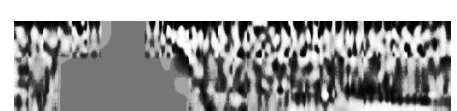

(b)

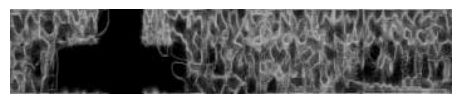

( c)
Figure 10. Iris Recognition Process

(a) Original Eye Image (b) Normalized and Enhanced Iris Pattern (c) Phase Congruency Representation

Table 2. Experimental Results : Iris Recognition

\begin{tabular}{|c|c|c|c|}
\hline Method & $\begin{array}{c}\text { Feature } \\
\text { Vector } \\
\text { Length } \\
\text { (Bits) }\end{array}$ & Classifier & $\begin{array}{c}\text { Recognition } \\
\text { Rate }\end{array}$ \\
\hline $\begin{array}{c}\text { Gabor } \\
\text { Wavelet }\end{array}$ & 2048 & $\begin{array}{c}\text { Hamming } \\
\text { Distance }\end{array}$ & $99 \%$ \\
\hline $\begin{array}{c}\text { Spatial } \\
\text { Filter }\end{array}$ & 1600 & $\begin{array}{c}\text { Euclidean } \\
\text { Distance }\end{array}$ & $98.3 \%$ \\
\hline $\begin{array}{c}\text { Phase } \\
\text { Congruency }\end{array}$ & 1024 & $\begin{array}{c}\text { Hamming } \\
\text { Distance }\end{array}$ & $93 \%$ \\
\hline
\end{tabular}

As compare to traditional Gabor wavelet based method, Phase Congruency based iris recognition method has slightly low recognition rate than Daugman's method. However, the iris capture devices in use are mostly exposed to the natural scene, phase congruency can provide better results under varying lightning conditions.

\section{CONCLUSION}

Phase congruency is low-level feature. Performance evaluation of CBIR system based on phase congruency shows that phase congruency shows great capability in extracting information from image. Recall of CBIR System based on phase congruency is 0.75 to 1 for different type of query images. Retrieval efficiency is 0.33 to 0.75 percent. The CBIR system performance is evaluated on small size database of 100 images and it needs to be tested on large size database. Experiments are also made to test the performance of iris feature extraction based on phase congruency. Traditional Gabor wavelet based method has slightly higher recognition rate as compared to phase congruency based iris recognition system. However phase congruency is more suitable to represent iris features for the eye images with an uneven illumination.

\section{REFERENCES}

[1] W. M. Smeulders, M. Worring, S.Santini, A.Gupta and R.Jain (2002), "Content based image retrieval at the end of early years", IEEE Transactions on Pattern Analysis and Machine Intelligence, vol. 22, pp 349-1379

[2] Colin C. Venteres and Dr. Matthew Cooper (2001), "A Review of Content-Based Image Retrieval Systems", International Conference on Image Processing.

[3] R. V. Patil, K. C. Jondhale (2009)," Content Based Image Retreival Based on Phase Congruency Via Log
Gabor Wavelet Filters", Proceedings of ICCVGIVP 2009. Nagpur, pp 84-85.

[4] Prakash K.S.S., RMD Sundaram (2007),” Combining Novel Features for Content Based Image Retrieval", Sixth EURASIP Conference focused on Speech and Image Processing, 373-376.

[5] Megha Agrawal and R. P. Maheshwari (2011), "Content Based Image Retrieval Based on Log Gabor Wavelet Transform", Advaned Materials Research, Vol. 403-408, pp. $871-878$

[6] Deng Y. , B. S. Manjunath (1997), "Content-based Search of Video Using Color, Texture, and Motion", Proc. Of IEEE International Conference on Image Processing, vol. 2, pp 534-537, Santra Barbara, CA

[7] Ryszard S. Chora's (2007), "Image Feature Extraction Techniques and Their Applications for CBIR and Biometrics and Systems", International Journal of Biology and Biomedical Engineering, vol. 1, pp. 6-16.

[8] P. S. Patil, S. R. Kolhe, R. V. Patil, P. M. Patil (2012) ,'The Comparison of Iris Recongition using Principal Component Analysis, Log Gabor and Gabor Wavelets", International Journal Of Computer Applications, Vol-43, No. 1., pp. 29-33

[9] B. J. Lei, Emile A. Hendriks, M.J.T. Reinders, "On Feature Extraction from Images", Technical Report, MCCWS Project, Information and Communication Theory Group, Tudeft

[10] R. V. Patil, K. C. Jondhale (2010)," Edge based technique to estimate number of clusters in k-means color image segmentation", IEEE Internatinal conference on Computer Sciene and Information Technology, Chengdu, China, vol. 2, pp. 117-121

[11] Jain, R. Bolle and S. Pankanti (1999) ," Biometrics: Personal Identification in a Networked Society", Kluwer Academic Publishers.

[12] A. Mansfield and J. Wayman (2002), "Best Practice Standards for Testing and Reporting on Biometric Device Performance," Nat'l Physical Laboratory of UK

[13] Xiaoyan Yuan, Pengfei Shi, "Iris Feature Extraction using 2 D Phase Congruency", Proceedings of the Third International Conference on Information Technology and Applications (ICITA'05),

[14] P Kovesi. (1999), "Image features from phase congruency.", Videre Journal of Computer Vision Research, 1(3), 1-27.

[15] P. Kovesi. (2003)," Phase congruency detects corners and edges.",In DICTA, Sydney.

[16] M. C. Morrone, Owens (1987), "Feature Detection From Local Energy", Pattern Recognition Letters, 310-313.

[17] Zheng Liu, R. Leganneire (2006), "On the Use of Phase Congruency to evaluate Image Similarity", IEEE International Conference on Accoustics, Speech and Signal Processing, 937-940.

[18] J. G. Daugman (1993),"High confidence visual recognition of persons by a test of statistical independence", IEEE Transactions on Pattern Analysis and Machine Intelligence, 15(11), 1148-1161. 
[19] J. Daugman (1994), "Biometric Personal identification System based on iris analysis", US patent no. 529160.

[20] J. Daugman (2003), "Demodulation by Complex valued wavelets for stochastic pattern recognition", International Journal of Wavelets, Multiresolution and Information Processing, 1(1), 1-17

[21] W. W. Boles and B. Boashash (1998), “A human wavelet transform", IEEE Transactions on Signal Processing, 46(4), 1185-1188.

[22] J. Daugman (2001), "Statistical Richness of Visual Phase Information: Update on Recognizing Persons by Iris Patterns," International Journal of Computer Vision, 45(1), 25-38.

[23] Hao Mang, Cuiping Xu (2006), "Iris Recognition Algorithms based on Gabor Wavelet Transforms", Proceedings of the 2006 IEEE International Conference on Mechatronics and Automation, Luoyang, China, 1785-1789

[24] Peng Fei Zhang, De-Sheng Li, Qi Wang (2004), "A Novel Iris Recognition Based On Feature Fusion", Proceedings of the Third International Conference on Machine Learning and Cybernetics, Shanghai, 36613665 .

[25] Henning Muller, Wolfang Muller, David Squire (1999), "Performance Evaluation in CBIR: Overview and Proposals", Technical Report, University of Geneva, Switezerland, 1999

[26] L.Ma, T. Tan, Y.Wang, and D. Zhang (2003), "Personal identification based on iris texture analysis," IEEE Transactions on Pattern Analysis and Machine Intelligence, 25(12), 1519-1533.
[27] L.Ma, T. Tan, Y. Wang, and D. Zhang (2004), "Efficient iris recognition by characterizing key local variations," IEEE Transactions on Image Processing, 13(6), 739-750.

[28] R. Johnson (1991), "Can Iris Patterns Be Used to Identify People?", Chemical and Laser Sciences Division LA-12331-PR, Los Alamos Nat'l Laboratory, Calif.

[29] L. Ma, Y. Wang, and T. Tan (2002), "Iris Recognition Based on Multichannel Gabor Filtering," Proc. Fifth Asian Conf. Computer Vision, 279-283.

[30] T. Lee (1996), "Image Representation Using 2D Gabor Wavelets," IEEE Trans. Pattern Analysis and Machine Intelligence, 18(10), 959-971.

[31] P. Belhumeur, J. Hespanha, and D. Kriegman (1997), "Eigenfaces vs. Fisherfaces: Recognition Using Class Specific Linear Projection," IEEE Trans. Pattern Analysis and Machine Intelligence, 19(7), 711-720.

[32] J. Zhang and T. Tan (2002), "Brief Review of Invariant Texture Analysis Methods," Pattern Recognition, 35(3), 735-747.

[33] V. Dorairaj and N. Schmid, and G. Fahmy (2005), "Performance Evaluation of Iris Based Recognition System Implementing PCA and ICA Techniques," in Proc. of the SPIE 2005 Symp. on Defense and Security, Conf. 5779, Orlando,.

[34] "CASIA Iris Image Database," http://www.sinobiometrics.com.

[35] D.M. Monro and D. Zhang (2005), "An Effective Human Iris Code with Low Complexity,” Proc. IEEE Int'l Conf. Image Processing, 3(3), 277-280.

[36] A.K. Jain, A. Ross, and S. Prabhakar (2004), "An Introduction to Biometric Recognition," IEEE Trans Circuits and Systems for VideoTechnology, 14, 4-20. 